\title{
Tratamento ortocirúrgico de paciente portador de deformidade dentofacial classe III: Relato de caso
}

\author{
Orthosurgical treatment of a patient with dentofacial deformity class III: Case report \\ Tratamiento ortosurgico de un paciente con deformidad dentofacial classe III: Reporte de caso
}

Recebido: 30/03/2021 | Revisado: 13/04/2021 | Aceito: 18/04/2021 | Publicado: 03/05/2021

\author{
Ana Maria Freitas Cavalcanti \\ ORCID: https://orcid.org/0000-0002-5354-0356 \\ Centro Universitário Facol, Brasil \\ E-mail: afreitascavalcanti@gmail.com \\ Lívia Mirelle Barbosa \\ ORCID: https://orcid.org/0000-0002-8992-2890 \\ Universidade de Pernambuco, Brasil \\ E-mail: dra.liviabarbosa@gmail.com \\ Sylvia Sampaio Peixoto \\ ORCID: https://orcid.org/0000-0002-2147-2627 \\ Universidade Federal de Pernambuco, Brasil \\ E-mail: sampaio_sylvia@outlook.com \\ Carolina Carollayne Clemente Dias Coelho \\ ORCID: https://orcid.org/0000-0002-9332-2513 \\ Universidade Federal de Pernambuco, Brasil \\ E-mail: Carolinadias92@hotmail.com \\ Jhony Herick Cavalcanti Nunes Negreiros \\ ORCID: https://orcid.org/0000-0002-3407-1021 \\ Universidade Federal de Pernambuco, Brasil \\ E-mail: jhonyherick@gmail.com \\ Priscilla Sarmento Pinto \\ ORCID: https://orcid.org/0000-0002-2376-4383 \\ Hospital da Restauração Governador Paulo Guerra, Brasil \\ E-mail: priscillasarmento@gmail.com \\ João Luiz Gomes Carneiro Monteiro \\ ORCID: https://orcid.org/0000-0001-6826-0798 \\ Universidade de Pernambuco, Brasil \\ E-mail: joaoluizgcm2@gmail.com \\ Maria Cecília Freire de Melo \\ ORCID: https://orcid.org/0000-0002-9592-4796 \\ Universidade Federal de Pernambuco, Brasil \\ E-mail: mceciliafreire@ hotmail.com \\ Alípio Miguel da Rocha Neto \\ ORCID: https://orcid.org/0000-0003-3488-0950 \\ Universidade de Pernambuco, Brasil \\ E-mail: alimiguel@outlook.com \\ Patrícia Mendonça Borba da Rocha \\ ORCID: https://orcid.org/0000-0002-1691-1779 \\ Universidade Federal de Pernambuco, Brasil \\ E-mail: patriciaborba@gmail.com \\ José Rodrigues Laureano Filho \\ ORCID: https://orcid.org/0000-0002-9645-2057 \\ Universidade de Pernambuco, Brasil \\ E-mail: laureano.filho@upe.br
}

\begin{abstract}
Resumo
A deformidade dentofacial é descrita como uma condição em que os ossos maxilares fogem da normalidade, onde a má oclusão existe e a estética facial é afetada. Esta condição acomete cerca de $20 \%$ da população mundial e requer um diagnóstico preciso. A cirurgia ortognática em combinação com o tratamento ortodôntico é a terapêutica de escolha para a correção das deformidades dentofaciais, devido a sua alta complexidade faz se necessário uma abordagem multidisciplinar para a realização de manejo terapêutico. Objetiva-se discutir neste artigo sobre o diagnóstico e tratamento das deformidades dentofaciais através de uma revisão de literatura e apresentação de um caso clínico. No caso reportado a paciente apresentava de deformidade dentofacial classe III e foi submetida ao tratamento ortodôntico e cirurgia ortognática planejada virtualmente com a utilização do software Dolphin 3D. Após o procedimento cirúrgico foi observada uma melhora significativa da harmonia facial e estabilidade oclusal. Tais resultados estão mantidos em acompanhamento pós-operatório por dois anos. O planejamento virtual em cirurgia ortognática vem se tornando cada vez mais importante, permitindo que os cirurgiões simulem múltiplas opções de movimentos maxilares
\end{abstract}


até que os melhores resultados sejam obtidos para o paciente.

Palavras-chave: Cirurgia ortognática; Anormalidades craniofaciais; Má oclusão dos dentes.

\begin{abstract}
Dentofacial deformity is described as a condition in which the jaw bones are out of normal, where malocclusion exists and facial aesthetics are affected. This condition affects about $20 \%$ of the world population and requires an accurate diagnosis. Orthognathic surgery in combination with orthodontic treatment is the treatment of choice for the correction of dentofacial deformities, due to its high complexity, a multidisciplinary approach is necessary to perform such treatment. This paper aims to discuss the diagnosis and treatment of dentofacial deformities through a literature review and presentation of a clinical case. In the case reported, the patient with class III dentofacial deformity underwent orthodontic treatment and orthognathic surgery planned virtually using the Dolphin 3D software. After the surgical procedure, a significant improvement in facial harmony and occlusal stability was observed. Such results are maintained in a two-year postoperative follow-up. Virtual planning in orthognathic surgery is becoming increasingly important, allowing surgeons to simulate multiple options of jaw movements until the best results are obtained for the patient.
\end{abstract}

Keywords: Orthognathic surgery; Craniofacial abnormalities; Malocclusion.

\title{
Resumen
}

La deformidad dentofacial se describe como una condición en la que los huesos de la mandíbula están fuera de lo normal, donde existe maloclusión y la estética facial se ve afectada. Esta condición afecta aproximadamente al $20 \%$ de la población mundial y requiere un diagnóstico preciso. La cirugía ortognática en combinación con el tratamiento de ortodoncia es el tratamiento de elección para la corrección de las deformidades dentofaciales, debido a su alta complejidad, es necesario un abordaje multidisciplinario para realizar el manejo terapéutico. El objetivo de este artículo es discutir el diagnóstico y tratamiento de las deformidades dentofaciales a través de la revisión de la literatura y la presentación de un caso clínico. En el caso reportado, el paciente presentaba una deformidad dentofacial clase III y se sometió a tratamiento de ortodoncia y cirugía ortognática planificada de forma virtual mediante el software Dolphin 3D. Después del procedimiento quirúrgico, se observó una mejora significativa en la armonía facial y la estabilidad oclusal. Estos resultados se mantienen en un seguimiento postoperatorio durante dos años. La planificación virtual en cirugía ortognática es cada vez más importante, ya que permite a los cirujanos simular múltiples opciones de movimientos de la mandíbula hasta obtener los mejores resultados para el paciente.

Palabras clave: Cirúgia ortognático; Anomalías craneofaciales; Maloclusión.

\section{Introdução}

As deformidades dentofaciais (DDF) podem ser descritas como as condições em que os ossos da face fogem da normalidade, onde a má oclusão existe e a aparência facial é afetada, essas deformidades podem ser mínimas como uma leve projeção do mento, ou extrema, como um excesso maxilar vertical severo ou uma microssomia hemifacial (Trench \& Araújo, 2015).

Estudos afirmam que a deformidade dentofacial acomete cerca de $20 \%$ da população mundial (Sadek \& Salem, 2007). Leite et al., (2004) analisaram, através de uma amostra com 180 pacientes brasileiros portadores de deformidades dentofaciais, oriundos de um centro de tratamento odontológico, que o tipo de deformidade mais comum foi a Classe III de Angle (53\%), Classe II (33\%) e Classe I (2\%).

A cirurgia ortognática combinada ao tratamento ortodôntico é a terapêutica de escolha para a correção das deformidades dentofaciais, possibilitando uma adequada correção das assimetrias faciais, permitindo o estabelecimento de um equilíbrio entre os dentes, ossos de sustentação e estruturas faciais vizinhas (Laureano Filho et al., 2003).

O principal objetivo e indicação da cirurgia ortognática é o de restabelecer um padrão facial considerado normal em pacientes adultos que terminaram o seu desenvolvimento craniofacial e apresentam grandes discrepâncias a nível ósseo (Estevão, 2012).

A correção cirúrgica das deformidades dentofaciais requer um planejamento abrangente para o posicionamento preciso de segmentos ósseos, assegurando que a oclusão dentária planejada será obtida (Ritto, 2012). 
A insatisfação com a estética facial é referida por muitos autores como o fator motivador mais regular na procura pela cirurgia ortognática. Estudos realizados encontraram anseios estéticos em $75 \%$ de seus pacientes, embora quase sempre acompanhados de anseios de ordem funcional. (Ambrizzi et al., 2007).

Além da correção das deformidades dentofaciais, a cirurgia ortognática é indicada para o tratamento de síndromes da ATM, com finalidade estética, tratamento de apneia do sono, ressecção de tumores da base do crânio ou como complemento na reabilitação oral em casos de acentuada reabsorção óssea alveolar, deficiências na fala e desordens psicossociais (Estevão, 2012).

Diante do exposto podemos observar que as deformidades dentofaciais são frequentes e necessitam de um tratamento multidisciplinar. O objetivo proposto por este trabalho é discutir sobre o diagnóstico e tratamento das deformidades dentofaciais, avaliar os benefícios do planejamento virtual na cirurgia ortognática e discutir o tratamento cirúrgico realizado no caso clínico de uma paciente portadora de deformidade dentofacial classe III, através de revisão da literatura.

\section{Materiais e Métodos}

Trata-se de um estudo de caso de características qualitativa e descritiva. De acordo com Pereira et al. (2018), pesquisas desta natureza caracterizam-se por elucidar um determinado assunto e estudá-lo minunciosamente. Este trabalho fundamenta-se em apresentar um caso clínico de tratamento ortocirúrgico de paciente com deformidade dentofacial classe III. Além disso, realizou-se uma revisão da literatura sobre este assunto, buscando artigos científicos nas bases de dados SCIELO e PUBMED, utilizando como descritores: orthognathic surgery, Craniofacial Abnormalities, Malocclusion. Com base nessa busca, foram selecionados artigos científicos com dados de grande relevância em relação ao tema proposto, como diagnóstico e tratamento.

\section{Considerações Éticas}

Os dados do paciente em questão foram coletados por meio do acesso direto ao prontuário e exames solicitados. Todos os princípios éticos descritos por Helsinque foram respeitados, objetivando proteger a vida, saúde, privacidade e dignidade do ser humano. A paciente assinou o Termo de Consentimento Livre Esclarecido (TCLE), termo do próprio hospital.

\section{Resultados}

\section{Deformidades Dentofaciais}

De acordo com Okazaki (1999), a deformidade dentofacial é definida como uma má oclusão esquelética que se caracteriza pela desarmonia entre os ossos maxilares da face. Essa condição pode estar evidente no nascimento ou manifestarse durante o crescimento e desenvolvimento, acarretando problemas funcionais, estéticos, psicológicos e sociais. A época para intervenção cirúrgica deve ocorrer durante ou após o crescimento ósseo completo (Trench \& Araújo, 2015).

Nas últimas décadas, o progresso técnico-científico nas áreas de Ortodontia e Cirurgia Ortognática permitiu variadas formas de planejamento integrado, com maior previsibilidade de resultados no tratamento de deformidades dentofaciais (Egermark, 2000).

A Deformidade Dentofacial é influenciada por diversos fatores, incluindo predisposição genética, fatores ambientais, trauma ou infecção facial na infância, cisto ou tumor, hábito parafuncional que causa má oclusão no desenvolvimento ósseo da face, hiperplasia condilar unilateral, hipoplasia mandibular, procedimentos cirúrgicos prévios ou distúrbio da articulação temporomandibular (Jung, Kim, Park \& Jung 2015). 
Segundo Van Spronsen (2010), o acometimento pode se apresentar em uma ou duas bases ósseas, nos planos vertical, horizontal e transversal, de maneira isolada ou combinada, causando diferentes tipos de deformidades.

$\mathrm{O}$ conhecimento das queixas estéticas e funcionais causadas pela deformidade e dos aspectos psicológicos envolvidos permite um contato direto entre profissionais e pacientes, fazendo-os chegar a um denominador comum, tendo como objetivo a satisfação do paciente, visando alcançar a harmonia facial, dentária e funcional com os resultados alcançados pelo tratamento ortodôntico e cirúrgico (Ambrizzi et al., 2007).

É notável que na maior parte dos casos, as alterações oclusais não são tratadas na infância, o que permite ao indivíduo alcançar a fase adulta ainda com a mesma alteração. Em outros casos, mesmo sendo realizado o tratamento ortodôntico na infância, este não é suficiente para resolução dos problemas dentofaciais do paciente. Nas demais situações, devido à insuficiência do tratamento ortodôntico na resolução das alterações esqueléticas, recorre-se à cirurgia ortognática. (Graziani, Garcia, Berretin \& Genaro, 2016).

Desta maneira, quando há modificações oclusais e faciais, associando-se às desproporções esqueléticas, a cirurgia ortognática é apontada como o principal tratamento de escolha, uma vez que sem a intervenção cirúrgica não há como alcançar mudanças das características das funções e da musculatura facial do paciente, o que é imprescindível para uma reabilitação completa. Devido a sua alta complexidade faz se necessário uma abordagem multidisciplinar para a realização do manejo terapêutico (Coutinho, Abath, Campos, Antunes \& Carvalho 2009).

De acordo com Garbin et al., (2010), as má oclusões são classificadas como:

\section{Classe I (neutroclusão)}

Variação do posicionamento dental no qual existe uma relação anteroposterior normal entre a maxila e a mandíbula. A crista triangular da cúspide mesiovestibular do primeiro molar permanente superior oclui no sulco mesiovestibular do primeiro molar permanente inferior. Sendo considerado Classe I o indivíduo que, que apresente a relação molar descrita, além de uma ou mais das seguintes características: giroversão, diastema, mordida cruzada, mordida aberta, mordida profunda e/ou atresia de arcada dentária.

\section{Classe II (distoclusão)}

Oclusopatia na qual se observa uma "relação distal" da mandíbula relativamente à maxila. O sulco mesiovestibular do primeiro molar permanente inferior oclui posteriormente à cúspide mesiovestibular do primeiro molar permanente superior, sendo:

- Divisão 1: distoclusão na qual os incisivos superiores estão tipicamente em labioversão.

- Divisão 2: distoclusão na qual os incisivos centrais superiores estão quase em sua posição normal anteroposteriormente ou apresentam uma leve linguoversão, enquanto os incisivos laterais superiores apresentam uma inclinação labial e mesial.

\section{Classe III (mesioclusão)}

Oclusopatia em que há relação "mesial" da mandíbula com a maxila. O sulco mesiovestibular do primeiro molar permanente inferior oclui anteriormente à cúspide mesiovestibular do primeiro molar permanente superior.

\section{Cirurgia Ortognática}

A cirurgia ortognática é o ramo da cirurgia bucomaxilofacial direcionada para o tratamento das deformidades dentofaciais, sendo realizada por uma equipe multidisciplinar que é organizada pelo cirurgião bucomaxilofacial e pelo 
ortodontista. Este tratamento visa a harmonia entre os tecidos moles e ósseos da face e as posições dentárias, também resolvendo problemas oclusais. (Guimaraes, Oliveira, Gomes e Souza, 2014).

Ortognática é uma palavra com origem nas palavras gregas "orthos" que significa reto e "gnathos" que corresponde a maxilares (Estevão, 2012). O termo cirurgia ortognática se refere ao "alinhamento dos ossos maxilares", visando corrigir desordens dentofaciais e maxilomandibulares. Muitos benefícios podem ser alcançados através da realização desse procedimento, como por exemplo, a melhoria nas funções do sistema estomatognático, e também a melhora da aparência e da estética facial, como consequência da melhora da relação maxilomandibular. (Nunes, 2017).

O desenvolvimento da cirurgia ortognática teve suas primeiras referências com as descrições e técnicas de reposicionamento dos segmentos esqueléticos faciais descritas desde o século XIX (Obwegeser, 2007). Nesta fase, as intervenções eram predominantemente limitadas à mandíbula, com a descrição de uma primeira osteotomia mandibular, realizada por Hullihen, em 1849. Esta primeira fase do desenvolvimento teve uma desaceleração com o início da primeira Guerra Mundial. (Panula, 2003).

Os principais motivos que levam os pacientes a procurar a cirurgia ortognática como tratamento incluem os problemas funcionais e dor facial, além da melhoria estética (Estevão, 2012). A insatisfação com a estética facial é dita por muitos autores como o agente motivador mais corrente na procura pela cirurgia ortognática (Ambrizzi et al., 2007).

Segundo Marques, Maniglia, Molina (2010), atualmente uma significativa parte da população apresenta algum desvio morfológico e/ou funcional do sistema estomatognático, acarretando assim um aumento da procura pelos tratamentos cirúrgicos para a correção de deformidades dentofaciais em nosso país, o que além do mais, cria também a necessidade de estudos de prevalência dessas desordens, para que se possa planejar e efetuar tratamentos de maneira adequada.

Em todos os casos torna-se de grande importância apresentar ao paciente as possibilidades de tratamento cirúrgico e ortodôntico, levando em conta a expectativa do resultado funcional e estético (Ferreira, Silva, Souza \& Freitas, 2011). Para Manganello, Silveira, Cappellette, Garducci, Lino (1998), o tratamento cirúrgico não elimina a fase ortodôntica pré-operatória, visto que ambas promovem ao paciente um resultado estético e funcional mais constante a longo prazo, minimizando a chance de recidiva.

A cirurgia ortognática pode apresentar algumas complicações durante a sua realização, sendo estas pouco frequentes (Nunes, 2017). Dentre elas, destacam-se a perda da sensibilidade orofacial, que pode ocorrer devido a osteotomias realizadas próximo aos ramos periféricos dos nervos maxilar e mandibular, podendo resultar em traumas dessas estruturas (Graziani, Garcia, Berretin \& Genaro, 2016). Coutinho e Moreno (2016) mencionam também hemorragia e mau posicionamento da maxila, e outras complicações mais raras como necrose isquêmica, fraturas não desejadas, fístulas, desvio de septo, sinusite maxilar, e danos aos sistemas nasal, lacrimal e ocular.

É imprescindível que, na primeira consulta, realize-se uma entrevista minuciosa com o paciente para discutir sua concepção dos problemas e objetivos de qualquer recurso terapêutico possível. O estado de saúde atual e problemas clínicos ou psicológicos que podem prejudicar o tratamento também devem ser analisados (Hupp, Ellis \& Tucker, 2009).

O diagnóstico se inicia com a realização de um exame clínico através da avaliação intrabucal, dando maior relevância à oclusão dentária, à pesquisa de distúrbios na articulação temporomandibular e à análise da estética facial. (Araújo, Gabrielli $\&$ Medeiros, 2007).

Nesta etapa é estabelecido o tipo de deformidade que o paciente apresenta, além de avaliar se é preciso ou não realizar exodontias, e que alterações ósseas serão realizadas na cirurgia. Definido o diagnóstico e plano de tratamento, o paciente é encaminhado ao ortodontista para que seja executada a ortodontia pré - cirúrgica. (Sant’ana \& Janson, 2003).

Arnett e Bergman (1993) esclareceram que o papel do Ortodontista no tratamento ortodôndico pré-cirúrgico é descompensar as posições dentárias inadequadas, eliminando as rotações dentárias, permitindo um correto posicionamento 
dentro da sua base óssea. O diálogo entre profissionais é de grande relevância, visto que a intenção do tratamento ortodôntico pré-cirúrgico em geral caminha de modo contrário ao de rotina ortodôntico, que normalmente procura disfarçar as assimetrias das bases ósseas. Nessa fase de ortodontia pré-cirúrgica os dentes precisam estar posicionados de modo correto em relação as estruturas ósseas, eliminando compensações, mantendo os dentes mais alinhados e estáveis, deixando a correção da interrelação das bases ósseas exclusivamente para a cirurgia, possibilitando a obtenção de excelentes resultados na fase póscirúrgica devolvendo assim a função. (Manfro, Cecconelo \& Frey, 2006).

Seja qual for o tipo de deformidade dentofacial onde cada situação tem suas características (ex.: deformidades Classe I, II ou III esquelética), o tratamento ortodôntico pode adotar a mesma ordem após o diagnóstico: fase pré-cirúrgica, fase transcirúrgica, fase pós-cirúrgica e fase de contenção (Mamani, 2013).

Para estabelecer um correto plano de tratamento em cirurgia ortognática, é de grande relevância a associação da avaliação clínica, da análise de modelos, análise facial, do estudo cefalométrico, além da cirurgia de modelos (Queiroz et al., 2010).

A análise facial deve ser realizada de acordo com as vistas frontal e de perfil do paciente, colocando-o em pé, na posição vertical e olhando para frente. $\mathrm{Na}$ vista frontal, verifica-se a presença de harmonia ou equilíbrio entre os três terços faciais (superior, médio e inferior). É necessário avaliar a simetria e harmonia entre ambos os lados da face, descartando as simples assimetrias que não façam parte da queixa principal do paciente (Araújo et al., 2007). Trata se de um método diagnóstico essencial, que auxilia no plano de tratamento, sendo visto como o principal elemento a ser explorado nos pacientes submetidos ao tratamento ortocirúrgico (Monteiro \& Vilella, 2007).

A análise cefalométrica é uma ferramenta de alta relevância na confirmação do diagnóstico, ainda que não seja o único resultado a ser avaliado. A harmonia facial e a oclusão precisam ser consideradas e acrescentadas ao exame cefalométrico, com finalidade de se obter diagnóstico preciso e o plano de tratamento adequado e eficaz (Queiroz et al., 2010).

Nota-se que a análise cefalométrica é essencial para a elaboração do diagnóstico e planejamento cirúrgico baseado no traçado predictivo permitindo prever os resultados a serem alcançados, os exames de imagem necessitam apresentar boa qualidade, com densidade e nitidez adequadas (Miloro et al., 2014; Albarakati, Kula \& Ghoneima, 2012).

$\mathrm{O}$ traçado predictivo é realizado de forma semelhante à convencional. Nessa etapa todo o planejamento precisa ser feito com as posições dentárias inicial e final acrescentada no traçado, entretanto em cores distintas (Faber, 2010).

Através do traçado predictivo cirúrgico é possível analisar as mudanças de perfil, programar exodontias e alterações ortodônticas necessárias. Esse traçado também pode ser aplicado para analisar o avanço do tratamento e a preservação do movimento cirúrgico na supervisão pós-operatória, permitindo ao paciente o entendimento do tratamento proposto e dos resultados previstos, tornando-o mais passível a colaborar com o tratamento (Queiroz et al., 2010).

A análise de modelos "permite diagnosticar todas as alterações intrabucais que devem ser corrigidas para a correta relação entre os maxilares e a base do crânio" (Papaleo et al., 2005).

Cirurgias ortognáticas bimaxilares necessitam da montagem dos modelos de estudo em articulador semiajustável de forma precisa, a fim de que a cirurgia de modelos represente a cirurgia real idealizada para o paciente. Isto possibilita a confecção das guias cirúrgicas intermediárias e finais imprescindíveis para o reposicionamento exato da maxila e da mandíbula (Gateno, Forrest \& Camp, 2001); (Wolford \& Aluisio, 2007).

A cirurgia dos modelos é uma etapa relevante do planejamento, necessária para o êxito das cirurgias ortognáticas bimaxilares, eliminando assim as limitações durante as fases de planejamento (análise facial, traçado de predição cirúrgica, cirurgia de modelos). A etapa laboratorial, sobretudo a habitual determinada a partir do modelo superior, quando realizada diretamente no articulador ASA, vem a ser um trabalho difícil e, geralmente, impreciso. Inicialmente, faz se necessário considerar a ação da gravidade sobre o modelo em movimentação, estabilizado apenas com cera. Muitas vezes existe um risco 
maior de imprecisão na obtenção das dimensões a serem alteradas e na preservação da estabilidade destas até a confecção do guia cirúrgico (goteira e splint) (Ellis, 1990).

"Os guias cirúrgicos gerados após o termino do planejamento são fundamentais para o posicionamento dos segmentos ósseos na posição desejada no pré-operatório" (Xia, Gateno \& Teichgraeber, 2005).

O planejamento virtual em cirurgia ortognática vem se tornando cada vez mais importantes. Alguns softwares são descritos para a realização do planejamento virtual em 3D dentre eles o SimPlant (Materialize, Leuven, Bélgica) e o Dolphin 3D (Dolphin Imaging and Management Solutions, Chatsworth, CA, EUA). Esses sistemas tem origens de desenvolvimento distintas. O SimPlant teve início por meio da cirurgia guiada em 3D na implantodontia, ao mesmo tempo que o Dolphin 3D teve sua origem através do traçado cefalométrico convencional bidimensional (2D) e planejamento lateral (Stokbro, Aagaard, Torkoy, Bell \& Thygesen, 2014).

Esse modelo de planejamento oferece novas medidas que permitem a visualização da relação entre os arcos dentários superior e inferior, além das estruturas ósseas de suporte, em um único modelo virtual. Esse modelo apresenta diferentes benefícios quando comparado ao planejamento convencional, incluindo: (1) uma avaliação de diagnóstico elaborada em um modelo virtual 3D; Esse método de diagnóstico permite identificar e avaliar a escala dentária, as deformidades dos ossos maxilares e outras assimetrias faciais que não seriam identificadas pelo exame físico, análise cefalométrica lateral 2D e modelos dentais de gesso montados em um articulador semi-ajustável (Swennen, Mollemans \& Schutyser, 2009; Baker, Goldstein \& Seruya, 2012; Ellis, 1990). (2) o planejamento virtual em 3D dá ao cirurgião a liberdade para simular diferentes procedimentos cirúrgicos, visando alcançar o resultado mais satisfatório possível para o paciente (3) o planejamento virtual em 3D favorece a avaliação e correção da relação central na articulação temporomandibular (Hsu et al., 2013).

\section{Caso Clínico}

Paciente do gênero feminino, 22 anos, compareceu ao serviço ambulatorial de cirurgia Bucomaxilofacial do Hospital São Marcos, onde se queixou de assimetria da face e dificuldade de mastigação. Durante o exame físico, foi observado desvio mandibular esquerdo, mordida cruzada anterior e no lado esquerdo (Figura 1). A linha média da maxila era igual à linha média da face. A linha média dos dentes inferiores e do mento apresentavam-se deslocados aproximadamente 5 e $7 \mathrm{~mm}$ respectivamente para esquerda. Diante da análise facial foi possível realizar o diagnóstico de deformidade dentofacial classe III associada à assimetria mandibular.

A paciente já estava em tratamento ortodôntico pré-operatório há cerca de 18 meses, no qual realizou-se o alinhamento e nivelamento dos dentes em sua base óssea. A paciente realizou tomografia computadorizada multislice completa da face, com corte de 1mm, cujas imagens foram salvas no formato DICOM (do inglês Digital Imaging and Communications in Medicine). Os modelos de gesso dos arcos dentários superior e inferior foram escaneados individualmente e em oclusão final, tendo suas imagens salvas no modelo STL (do inglês Stereolithography). Essas imagens foram exportadas para o software de planejamento virtual (Dolphin 3D) para manipulação virtual e simulação dos movimentos cirúrgicos necessários para a correção da deformidade dentofacial. O planejamento realizado baseou-se na rotação do plano oclusal com giro horário do complexo maxilomandibular e correção do desvio mandibular (Figura 2). Simulou-se a osteotomia Le Fort I e impactação posterior da maxila, a partir desse movimento foi gerado um guia oclusal intermediário. Também foi simulada a osteotomia sagital de mandíbula virtualmente, permitindo a movimentação mandibular de recuo e desvio para direita, estabilizando a oclusão e corrigindo a assimetria. O planejamento também incluiu a mentoplastia de avanço. Com base no movimento 
mandibular o programa gerou o guia oclusal final. As imagens dos guias foram salvas no formato STL e impressas em resina acrílica.

Durante a fase cirúrgica, realizou-se osteotomia Le Fort I. Em concordância com o planejamento, o segmento da maxila mobilizado foi girado no sentido horário e reposicionado com base no guia intermediário, após o bloqueio maxilomandibular com fio de aço $\mathrm{n}^{\circ} 0$ realizou-se a fixação interna da maxila com placas e parafusos do sistema 1,5 mm. Sendo utilizada uma placa Lindorf no pilar canino e uma placa em L no pilar zigomático-maxilar em cada maxila. Em seguida realizou-se a liberação do bloqueio maxilomandibular e as osteotomias mandibulares. O segmento distal da mandíbula foi virado para a direita e colocado na oclusão pretendida, utilizando-se o guia oclusal final. Em seguida foi realizada a osteossíntese com duas placas retas de titânio, do sistema $2.0 \mathrm{~mm}$ no corpo mandibular bilateralmente. A etapa de mentoplastia incluiu a osteotomia do mento, seu reposicionamento conforme o planejamento virtual e fixação com placa em X do sistema $2.0 \mathrm{~mm}$. A ortodontia pós-cirúrgica foi reiniciada 2 meses após a cirurgia. $\mathrm{O}$ alinhamento final das arcadas e o encaixe oclusal foram exercidos no decorrer dos 6 meses subsequentes. Houve uma melhora significativa da harmonia facial após o procedimento cirúrgico. A oclusão apresenta-se equilibrada e a paciente está satisfeita com o resultado após dois anos de acompanhamento. A Figura 3 corresponde ao aspecto inicial e do pós-operatório de um mês, evidenciando o estabelecimento da harmonia facial e boa oclusão.

Figura 1. A. Aspecto facial em vista frontal evidenciando o desvio mandibular para o lado esquerdo. B. Vista oclusal evidenciando a mordida cruzada anterior e do lado esquerdo.

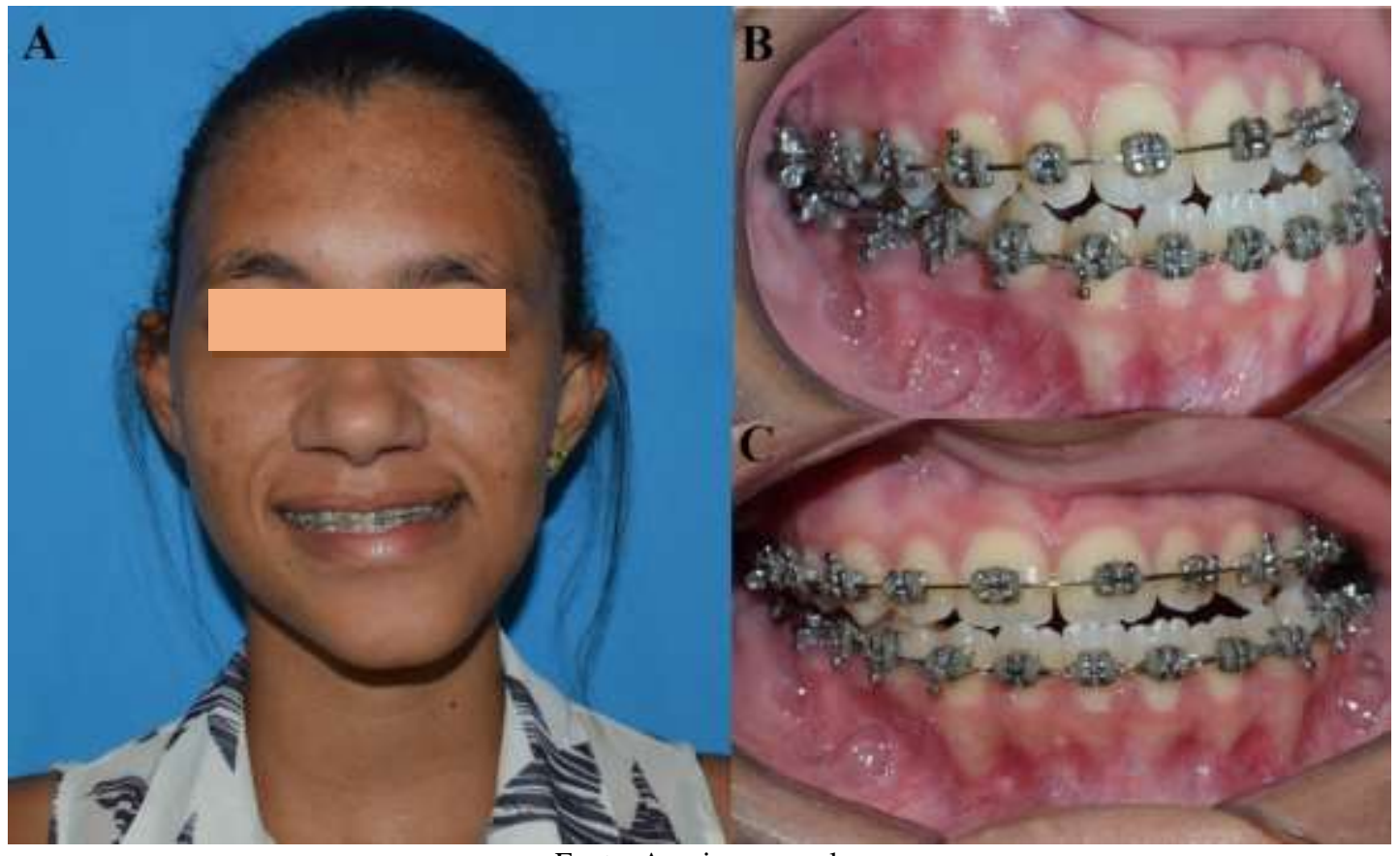

Fonte: Arquivo pessoal. 
Figura 2. Imagem do Planejamento virtual da cirurgia ortognática obtida do programa Dolphin 3D. A e B. Aspecto inicial da paciente com a simulação das osteotomias, em vista frontal e de perfil respectivamente. C e D. Aspecto final após simulação do giro horário do giro horário do complexo maxilomandibular e mentoplastia de avanço, em vista frontal e de perfil respectivamente.

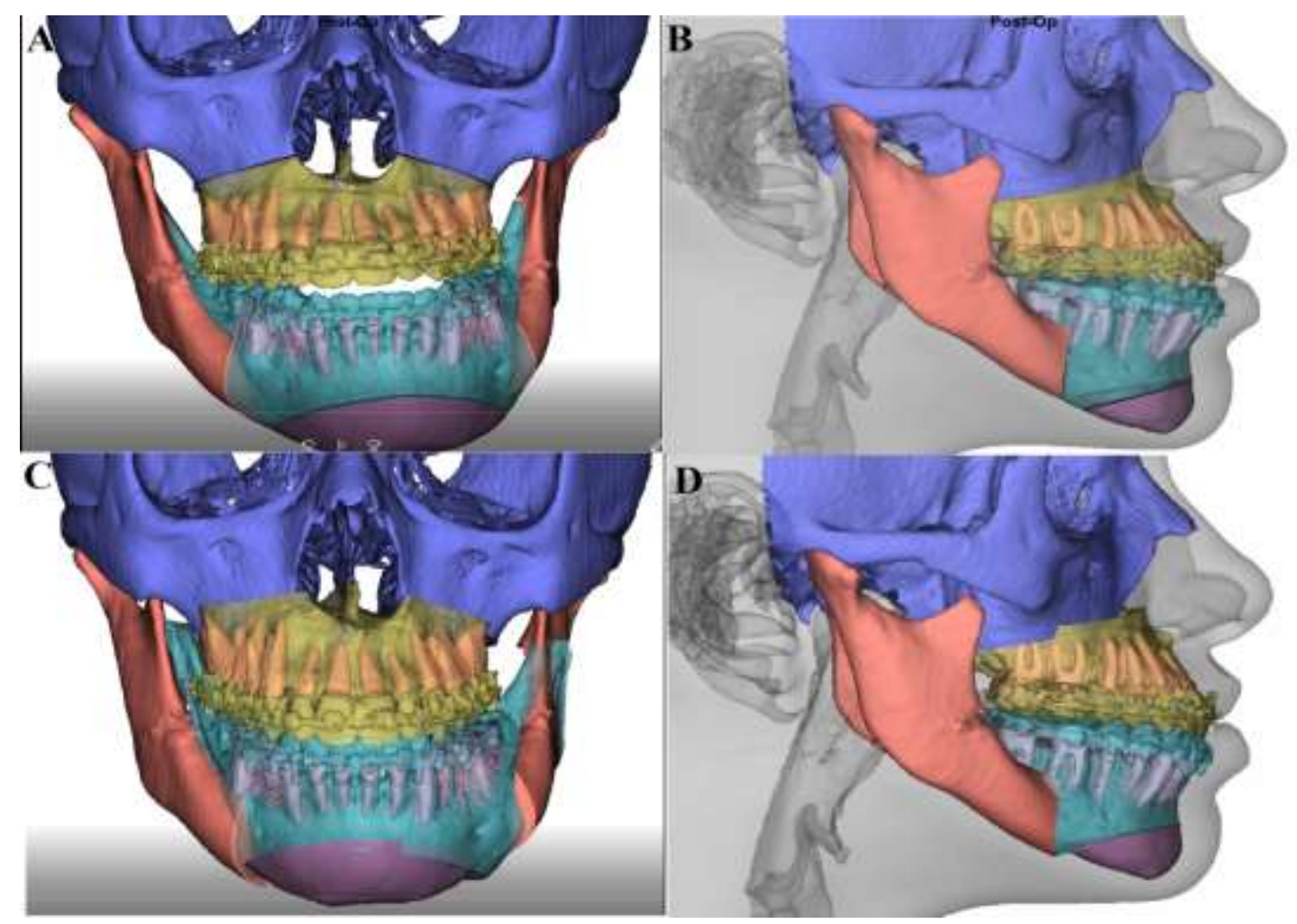

Fonte: Arquivo pessoal. 
Figura 3. Aspecto inicial e do pós-operatório de um mês, evidenciando o estabelecimento da harmonia facial e boa oclusão.

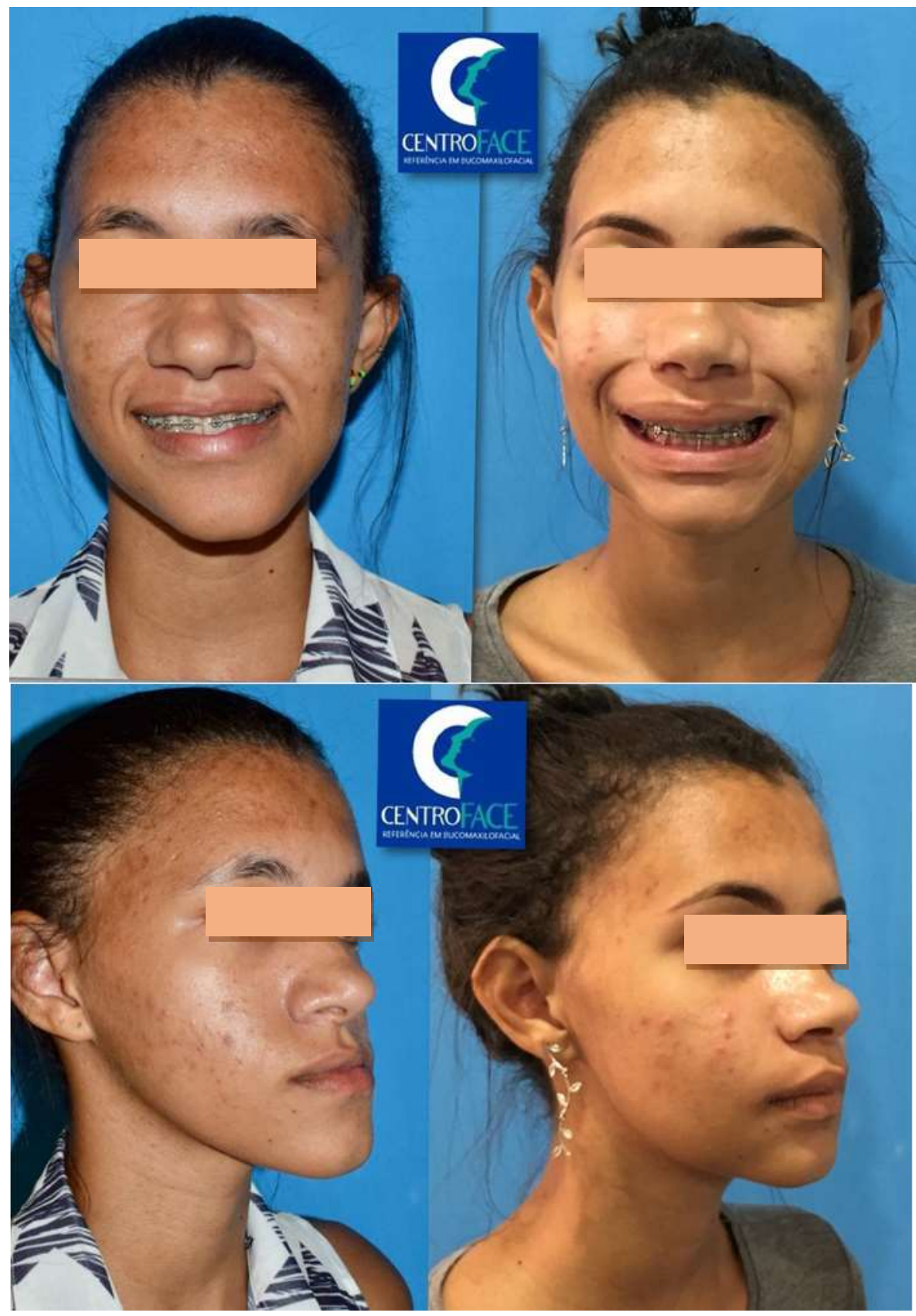

Fonte: Arquivo pessoal.

\section{Discussão}

O caso relatado acima foi de uma paciente com deformidade dentofacial classe III, associada a desvio mandibular esquerdo, mordida cruzada anterior e no lado esquerdo. A paciente já estava em tratamento ortodôntico pré-operatório há cerca de 18 meses, no qual realizou-se o alinhamento e nivelamento dos dentes em sua base óssea.

O tratamento desse tipo de deformidade envolve a reabilitação cirúrgica, por meio da cirurgia ortognática, e a sua importância não se limita apenas a correção oclusal, mas também da melhora na estética da face. Isso quer dizer que os aspectos psicológicos e sociais se encontram diretamente associados a este tipo de tratamento, pois a estética facial interfere no desenvolvimento da imagem corporal, identidade e autoestima (Nicodemo, Pereira \& Ferreira, 2007). 
O planejamento realizado baseou-se na rotação do plano oclusal com giro horário do complexo maxilomandibular e correção do desvio mandibular, onde por meio do software Doplhin 3D foi feita toda a manipulação virtual e simulação dos movimentos cirúrgicos necessários.

O planejamento virtual é executado em um modelo virtual composto (crânio composto) constituído baseado na tomografia e nos modelos dentários escaneados, permitindo um a projeção de um modelo aproximado da realidade. Além disto, os softwares de planejamento virtual 3D possibilitam ao cirurgião realizar osteotomias digitais e prever o efeito dos movimentos planejados nos ossos e tecidos moles do paciente (De Barros Dias et al., 2016).

A cirurgia ortognática comum geralmente não altera a inclinação da superfície oclusal. Quando uma alteração é necessária, a rotação em geral é realizada no sentido horário. Ao se deparar com pacientes portadores de malformações anteroposterior graves, o giro do complexo maxilomandibular é um a boa opção de tratamento, embora, ao mesmo tempo, haja pouca alteração no nível de sobreposição horizontal. Quando a superfície oclusal muda, é possível alcançar mudanças consideráveis na projeção do queixo, mesmo que a sobreposição horizontal seja pequena (Pary Martin \& Richard, 2008). Durante a fase cirúrgica, realizou-se osteotomia Le Fort I. Em concordância com o planejamento, o segmento da maxila mobilizado foi girado no sentido horário e reposicionado com base no guia intermediário.

$\mathrm{O}$ avanço maxilomandibular com giro no sentido horário preserva a oclusão dental e proporciona uma maior proeminência do terço inferior da face, desta forma alonga o comprimento entre o mento e o pescoço promovendo uma maior sustentação aos tecidos moles da face, resultando em um efeito de "lifting" facial, além de preencher a área do osso zigomático. O padrão da face passa a ter um maior equilíbrio, tornando-se mesofacial (De Lima Stevão, 2009).

A ortodontia pós-cirúrgica foi reiniciada 2 meses após a cirurgia e finalizada nos 6 meses subsequentes. A paciente apresentou uma melhora significativa da harmonia facial após o procedimento cirúrgico. A oclusão apresenta-se equilibrada e a mesma está satisfeita com o resultado após dois anos de acompanhamento.

\section{Considerações Finais}

A deformidade dentofacial é uma condição que afeta significativa parte da população, acarretando problemas estéticos, psicológicos e sociais, onde sua correção é um verdadeiro desafio para os cirurgiões bucomaxilofaciais. A cirurgia ortognática em combinação com a ortodontia é o tratamento de escolha para a correção das deformidades dentofaciais, procedimento este, que devolve a harmonia facial e melhora a qualidade de vida dos pacientes. O tratamento ortocirúrgico deve ser realizado da melhor forma, para que o resultado final supere as expectativas do paciente. Um planejamento précirurgico bem executado é essencial, assim como um bom planejamento virtual para que um resultado satisfatório seja obtido.

Futuros trabalhos são necessários para aprofundar o conhecimento sobre o assunto.

\section{Referências}

Albarakati, S. F., Kula, K. S., \& Ghoneima, A. A. (2012). The reliability and reproducibility of cephalometric measurements: a comparison of conventional and digital methods. Dentomaxillofacial Radiology, 41(1), 11-17.

Ambrizzi, D. R., Franz, S. A., Pereira Filho, V. A., Gabrielli, M. A. C., Gimenez, C. M. M., \& Bertroz, F. A. (2007). Avaliação das queixas estético-funcionais em pacientes portadores de deformidades dentofaciais. Revista Dental Press de Ortodontia e Ortopedia Facial, 12(5), 63-70.

Araújo, A. M., Gabrielli, M. F. R., \& Medeiros, P. J. (2007). Cirurgia Ortognática: O tratamento das deformidades dentofaciais. Aspectos atuais da Cirurgia e Traumatologia Bucomaxilofacial. (1a ed., pp.271-301). São Paulo: Editora Santos.

Arnett G. W., \& Bergman R. T. (1993). Facial keys to orthodontic diagnosis and treatment planning - part I. American Journal of Orthodontics and Dentofacial Orthopedics, 103(4), 299-312.

Baker, S. B., Goldstein, J. A., \& Seruya, M. (2012). Outcomes in computer-assisted surgical simulation for orthognathic surgery. J Craniofac Surg 23(2), 509513. 
Coutinho, Eduardo de Freitas; Moreno, Tatiana Ferreira (2016). Complicações relacionadas à osteotomia le fort I total em cirurgia ortognática de maxila. Revista da ACBO, 5(1), 1-16.

Coutinho, T. A., Abath, M. D. B., Campos, G. J. D. L., Antunes, A. A., \& Carvalho, R. W. F. D. (2009). Adaptações do sistema estomatognático em indivíduos com desproporções maxilo-mandibulares: revisão da literatura. Revista da Sociedade Brasileira de Fonoaudiologia 14(2), 275-279.

De Barros Dias, B. S., Pascual, C., Scheneider, T., Guedes, F. P., Seabra, R., \& Filho, L. C. (2016) Planejamento virtual: uma realidade no tratamento das deformidades dentofaciais. Revista Clínica de Ortodontia Dental Press 15(3), 83-105.

Stevão, É. L. L. (2006). Ortodontia e Cirurgia Ortognática associadas à Rinoplastia - Uma Realidade Atual. Momento \& Perspectivas em Saúde, Porto Alegre - RS, 19(1), 9-19.

Egermark, I., Blomqvisit, J. E., Cromvik, U., \& Isaksson, S. (2000). Temporomandibular dysfunction in patients treated with orthodontics in combination with orthognathic surgery. The European Journal of Orthodontics, 22(5), 537-544.

Ellis III, E. (1990). Accuracy of model surgery: evaluation of an old technique and introduction of a new one. J. Oral Maxillofac. Surg., 48(11),1161-1167.

Estevão, V. S. D. S. V. (2012). Cirurgia Ortognática: Correlação das deformidades dentofaciais. Monografia, Faculdade de Medicina da Universidade do Porto, Porto, Portugal.

Faber, J. (2010). Benefício Antecipado: uma nova abordagem para o tratamento com cirurgia ortognática que elimina o preparo ortodôntico convencional. Dental Press Journal of Orthodontics, 15(1), 144-157.

Ferreira, A. A., Silva, L. C., Souza, S. L., \& Freitas, A. (2011) Tratamento das deformidades maxilofaciais. Rev Bras Cir Craniomaxilofac, $12(3)$, 129-132.

Garbin, A. J., Perin, P. C. P., Garbin, C. A. S., \& Lolli, L. F. (2010). Prevalência de oclusopatias e comparação entre a Classificação de Angle e o Índice de Estética Dentária em escolares do interior do estado de São Paulo-Brasil. Dental Press Journal of Orthodontics, 15(4), 94-102.

Gateno J., Forrest K. K., \& Camp. B. A. (2001). Comparison of 3 methods of face-bow transfer recording: implications for orthognathic surgery. $J$ Oral Maxillofac Surg, 59(6), 635-40.

Graziani, A. F., Garcia, C. F. S., Berretin, F. G., \& Genaro, K. F. (2016). Orthognathic surgery effect of orofacial sensitivity in individuals with cleft lip a palate. Rev. CEFAC. 18(3), 581-587.

Guimaraes Filho, R., Oliveira Junior, E. C., Gomes, T. R. M., \& Souza, T. D. A. D. (2014). Calidad de vida en pacientes sometidos cirugía ortognática: salud bucal y autoestima. Psicologia: Ciência e Profissão, 34(1), 242-251.

Hsu, S. S., Gateno, J., Bell, R. B., Hirsch, D. L., Markiewicz, M. R., Teichgraeber, J. F., \& Xia, J. J. (2013). Accuracy of a computer-aided surgicalsimulation protocol for orthognathic surgery: a prospective multicenter study. J Oral Maxillofac Surg, 71, 128-42.

Hupp, J. R., Ellis III, E., \& Tucker, M. R. (2009). Correção das deformidades dentofaciais. In: Tucker, Myron R.; Farrel, Brian B.; Farrel, Bart C. Cirurgia Oral e Maxilofacial Contemporânea. (5a ed.), 515-557. Elsevier.

Jung, H. D., Kim, S. Y., Park, H. S., \& Jung, Y. S. (2015). Orthognathic surgery and temporomandibular joint symptoms. Maxillofacial plastic and reconstructive surgery, $37(1), 14$.

Laureano Filho, J. R., Cypriano, R. V., Moraes, R. P. A., \& Freitas, M. Q. (2003). Avanço maxilar: descrição da técnica e relato de caso clínico. Rev Cir Traumatol Buco-maxilo-fac, 3(3), 33-40

Leite, P., Camarini, E., Filho, L., Pavan, A., Farah, G., \& Silva, M. (2004). Estudo epidemiológico das deformidades dentofaciais de Maringá. Pesquisa Brasileira Odontopediatria Clínica Integrada (João Pessoa), 4(3),217-220.

Mamani, M. H. (2013). Preparo ortodôntico em casos de cirurgia ortognática. Trabalho de Conclusão de Curso (especialização), Faculdade Estadual de Campinas, São Paulo, Brasil.

Manfro, R., Cecconelo, R., \& Frey, M. A. T. (2006). Inter-relação cirurgia ortognática, ortodontia e implantodontia: apresentação de caso clínico. Rev. Innovations Journal, 1, 39-42.

Manganello, L. C. S., Silveira, M. E., Cappellette, M., Garducci, M., \& Lino, A. P. (1998) Cirurgia ortognática e ortodontia. Editora Santos.

Marques, C. G., Maniglia, J. V., \& Molina, F. D. (2010). Perfil do Serviço de Cirurgia Ortognática de uma escola médica. Brazilian Journal of Otorhinolaryngology,76(5), 600-604.

Miloro, M., Borba, A. M., Ribeiro Junior, O., Naclerio Homem, M. G., \& Jungner, M. (2014). Is there consistency in cephalometric landmark identification amongst oral and maxillofacial surgeons? International journal of oral and maxillofacial surgery, 43(4), 445-453.

Monteiro, M. R. G. M.; \& Vilella, O. V. (2007). Tratamento ortodôntico-cirúrgico de maloclusão de Classe II Divisão I. Rev. bras. odontol, $137-140$.

Nicodemo, D., Pereira, M. D., \& Ferreira, L. M. (2007). Cirurgia Ortognática: abordagem psicossocial em pacientes Classe III de Angle submetidos à correção cirúrgica da deformidade dentofacial. Revista Dental Press de Ortodontia e Ortopedia Facial, 12(5), 46-54.

Nunes, J. D. S. (2017) Indicações e Prognósticos em Cirurgia Ortognática. Monografia, Faculdade de Macapá, Amapá, Brasil.

Obwergeser, H. L. (2007). Orthognathic surgery and a tale of how three procedures came to be: a letter to the next generations of surgeons. Clinics in plastic surgery, 34(3), 331-355.

Okazaki, L. K. (1991). Quando indicar uma cirurgia ortognática. In: Araújo A, editor. Cirurgia ortognática. (pp.7-18). Editora Santos. 
Research, Society and Development, v. 10, n. 5, e18510514451, 2021

(CC BY 4.0) | ISSN 2525-3409 | DOI: hittp://dx.doi.org/10.33448/rsd-v10i5.14451

Panula, K. (2003). Correction of dentofacial deformities with orthognathic surgery. Int J Adult Orthognath Orthod Surg, 17, 297-306.

Papaleo, E. C., Schneider, L. E., Crusius, K. C., De Souza Nunes, L. S., Vargas, I. A., \& Hernandez, P. A. G. (2005). Aspectos relevantes na análise de modelos aplicada à cirurgia ortognática. Stomatos, 11(20), 37-41.

Pary, A. H., Martins, H., \& Richard, G. M. (2008). Tratamento de micrognatia mandibular severa com rotação do complexo maxilomandibular. Rev. Clin. Odont. Dental Press, 7(5), 64-70.

Queiroz, T. P., Gulinelli, J. L., Souza, F. Á., Zanetti, L. S. D. S., Magro Filho, O., Garcia Junior, I. R., \& Vieira, E. H. (2010) Análise da fidelidade do traçado predictivo em pacientes submetidos à cirurgia ortognática em mandíbula. Dental Press Journal of Orthodontics,15(4), 117-123.

Ritto, F. G. (2012). Precisão do posicionamento maxilar em cirurgias bimaxilares utilizando sequência cirúrgica convencional e sequência invertida. Tese de Doutorado, Faculdade de Odontologia de Piracicaba UNICAMP, São Paulo, Brasil.

Sadek, H., \& Salen, G. (2007). Psychological aspects of orthognathic surgery and its effect on quality of life in Egyptian patients. La Revue de Santé de la Méditerranée Orientale, 13(1),150-159.

Sant'ana, E., \& Janson, M. D. R. P. (2003) Ortodontia e Cirurgia Ortognática: do planejamento à finalização. Revista Dental Press de Ortodontia e Ortopedia Facial, 8(3), 1-11.

Stokbro, K., Aagaard, E., Torkoy, P., Bell, R. B., \& Thygesen, T. (2014). Virtual planning in orthognathic surgery. International Journal of Oral and Maxillofacial Surgery, 43(8), 957-965.

Swennen, G. R., Mollemans, W., \& Schutyser, F. (2009).Three-dimensional treatment planning of orthognathic surgery in the era of virtual imaging. J Oral Maxillofac Surg, 67, 2080-92.

Trench, J. D. A., \& Araujo, R. P. C. D. Deformidades dentofaciais: características miofuncionais orofaciais. (2015). Revista CEFAC, 17(4), $1202-1214$.

Van Spronsen, P. H. (2010). Long-face craniofacial morphology: Cause or effect of weak masticatory musculature? In: Seminars in Orthodontics. WB Saunders, 99-117.

Wolford, L. M., \& Aluisio, G. A. (2007). Simple and Accurate Method for Mounting Models in Orthognathic Surgery. J Oral Maxillofac Surg.,65(7), 1406-9.

Xia, J. J., Gateno, J., \& Teichgraeber, J. F. (2005). Three-dimensional computer-aided surgical simulation for maxillofacial surgery. Atlas of the oral and maxillofacial surgery clinics of North America, 13(1), 25-39. 